\title{
BOUNDARY CONTROLLABILITY IN PROBLEMS OF TRANSMISSION FOR A CLASS OF SECOND ORDER HYPERBOLIC SYSTEMS
}

\author{
JOHN E. LAGNESE
}

\begin{abstract}
We consider transmission problems for general second order linear hyperbolic systems having piecewise constant coefficients in a bounded, open connected set with smooth boundary and controlled through the Dirichlet boundary condition. It is proved that such a system is exactly controllable in an appropriate function space provided the interfaces where the coefficients have a jump discontinuity are all star-shaped with respect to one and the same point and the coefficients satisfy a certain monotonicity condition.
\end{abstract}

\section{INTRODUCTION AND MAIN RESULTS}

Let $\Omega, \Omega_{1}$ be bounded, open, connected sets in $\mathbb{R}^{n}$ with smooth boundaries $\Gamma$ and $\Gamma_{1}$, respectively, such that $\bar{\Omega}_{1} \subset \Omega$, and set $\Omega_{2}=\Omega \backslash \bar{\Omega}_{1}$, whose boundary is $\Gamma_{2}=\Gamma \cup \Gamma_{1}$. Let $m$ and $n$ be positive integers. For $i, k=$ $1, \ldots, m$, for $j, \ell=1, \ldots, n$ and for $\alpha=1,2$, let $a_{\alpha}^{i j k \ell}$ be real numbers having the symmetry

$$
a_{\alpha}^{i j k \ell}=a_{\alpha}^{k \ell i j}, \quad \alpha=1,2 ; i, k=1, \ldots, m ; j, \ell=1, \ldots, n .
$$

When $m=n$ we further assume that

$$
a_{\alpha}^{i j k \ell}=a_{\alpha}^{j i k \ell}=a_{\alpha}^{i j \ell k}, \quad \alpha=1,2 ; i, j, k, \ell=1, \ldots, n .
$$

The $a_{\alpha}^{i j k \ell}$ are also assumed to satisfy the following ellipticity condition:

$$
\begin{aligned}
& \text { if } m \neq n \text { then } \quad a_{\alpha}^{i j k \ell} \xi_{i j} \xi_{k \ell} \geq c_{0} \xi_{i j} \xi_{i j}, \quad c_{0}>0, \forall\left(\xi_{i j}\right) \in \mathbb{R}^{m \times n} ; \\
& \text { if } m=n \text { then } a_{\alpha}^{i j k \ell} \xi_{i j} \xi_{k \ell} \geq c_{0} \xi_{i j} \xi_{i j}, \quad c_{0}>0, \forall\left(\xi_{i j}\right) \in S^{n},
\end{aligned}
$$

where $S^{n}$ denotes the set of all real symmetric $n \times n$ matrices. In (1.3), (1.4) and in what follows, a repeated Roman index indicates summation with respect to that index over an appropriate range (either 1 to $m$ or 1 to $n$, as the context suggests), unless otherwise noted.

Fix $T>0$ and let

$$
\mathbf{W}_{1}=\left(\begin{array}{c}
w_{11} \\
\vdots \\
w_{1 m}
\end{array}\right), \quad \mathbf{W}_{2}=\left(\begin{array}{c}
w_{21} \\
\vdots \\
w_{2 m}
\end{array}\right)
$$

Department of Mathematics, Georgetown University, Washington, DC 20057 USA. Received by the journal April 15, 1997. Accepted for publication October 13, 1997.

(C) Société de Mathématiques Appliquées et Industrielles. Typeset by ATEX. 
be vector functions defined on the cylinders $Q_{1}=\Omega_{1} \times(0, T)$ and $Q_{2}=$ $\Omega_{2} \times(0, T)$, respectively. We set $\Sigma=\Gamma \times(0, T)$ and $\Sigma_{1}=\Gamma_{1} \times(0, T)$ and consider the following problem of transmission:

$$
\begin{cases}\ddot{w}_{1 i}-a_{1}^{i j k \ell} w_{1 k, \ell j}=0 & \text { in } Q_{1}, \\ \ddot{w}_{2 i}-a_{2}^{i j k \ell} w_{2 k, \ell j}=0 & \text { in } Q_{2}, \quad i=1, \ldots, m,\end{cases}
$$

where ${ }^{\circ}=\partial / \partial t$ and where a subscript following a comma indicates differentiation with respect to the corresponding spatial variable;

$$
\begin{gathered}
\mathbf{W}_{2}=\mathbf{U} \quad \text { on } \Sigma, \\
w_{1 i}=w_{2 i}, \quad a_{1}^{i j k \ell} w_{1 k, \ell} \nu_{j}=a_{2}^{i j k \ell} w_{2 k, \ell} \nu_{j} \quad \text { on } \Sigma_{1}, \quad i=1, \ldots, m,
\end{gathered}
$$

where $\nu=\left(\nu_{1}, \ldots, \nu_{n}\right)$ is the unit normal to $\Gamma_{1}$ pointing into $\Omega_{1}$ (and towards the exterior of $\Omega_{2}$ ),

$$
\left.\mathbf{W}_{\alpha}\right|_{t=0}=\left.\dot{\mathbf{W}}_{\alpha}\right|_{t=0}=0 \quad \text { in } \Omega_{\alpha}, \alpha=1,2 .
$$

The function $\mathbf{U}$ in (1.6) is viewed as a control function which is chosen to effect the evolution of the dynamics. More precisely, we consider below the reachability problem, which is to determine the set

$$
\mathcal{R}_{T}=\left\{\left(\mathbf{W}_{1}(T), \mathbf{W}_{2}(T), \dot{\mathbf{W}}_{1}(T), \dot{\mathbf{W}}_{2}(T)\right): \mathbf{U} \in \mathcal{U}\right\}
$$

where $\mathcal{U}$ is an appropriate space of controls.

The question of boundary controllability in problems of transmission has been considered by several authors. In particular, J.-L. Lions considered the system (1.5) - (1.8) in the special case of two wave equations, that is, he considered the problem

$$
\begin{cases}\ddot{w}_{1}-a_{1} \Delta w_{1}=0 & \text { in } Q_{1}, \\ \ddot{w}_{2}-a_{2} \Delta w_{2}=0 & \text { in } Q_{2},\end{cases}
$$

where $a_{\alpha}>0$ and $\Delta$ is the ordinary Laplacian in $\mathbb{R}^{n}$,

$$
\begin{gathered}
w_{2}=u \quad \text { on } \Sigma, \\
w_{1}=w_{2}, \quad a_{1} \frac{\partial w_{1}}{\partial \nu}=a_{2} \frac{\partial w_{2}}{\partial \nu} \quad \text { on } \Sigma_{1}, \\
\left.w_{\alpha}\right|_{t=0}=\left.\dot{w}_{\alpha}\right|_{t=0}=0 \quad \text { in } \Omega_{\alpha}, \alpha=1,2 .
\end{gathered}
$$

For this problem the following result is proved in [5, Chapter VI].

THEOREM 1.1. Assume that $\Gamma_{1}$ is star-shaped with respect to some point $x_{0} \in \Omega_{1}$ and set $\Gamma\left(x_{0}\right)=\left\{x \in \Gamma \mid\left(x-x_{0}\right) \cdot \nu>0\right\}, \Sigma\left(x_{0}\right)=\Gamma\left(x_{0}\right) \times(0, T)$, where $\nu$ is the unit outer normal to $\Gamma$. Let

$$
\mathcal{R}_{T}=\left\{\left(w_{1}(T), w_{2}(T), \dot{w}_{1}(T), \dot{w}_{2}(T)\right): u \in L^{2}(\Sigma), u=0 \text { on } \Sigma \backslash \Sigma\left(x_{0}\right)\right\} .
$$

If $a_{1}>a_{2}$ and $T>T\left(x_{0}\right):=2 R\left(x_{0}\right) / \sqrt{a_{2}}$, where $R\left(x_{0}\right)=\max _{x \in \bar{\Omega}_{2}}\left|x-x_{0}\right|$, then $\mathcal{R}_{T}=H \times V^{\prime}$ where

$$
\begin{gathered}
H=L^{2}\left(\Omega_{1}\right) \times L^{2}\left(\Omega_{2}\right), \\
V=\left\{\left(\varphi_{1}, \varphi_{2}\right) \in H^{1}\left(\Omega_{1}\right) \times H^{1}\left(\Omega_{2}\right):\left.\varphi_{2}\right|_{\Gamma}=0,\left.\varphi_{1}\right|_{\Gamma_{1}}=\left.\varphi_{2}\right|_{\Gamma_{1}}\right\} .
\end{gathered}
$$


The main purpose of this paper is prove the following generalization of Theorem 1.1 to the system (1.5) - (1.8). Let us define

$$
\left|A_{2}\right|=\left\{\begin{array}{cl}
\inf _{0 \neq\left(\xi_{i j}\right) \in \mathbb{R}^{m \times n}} \frac{a_{2}^{i j k \ell} \xi_{k l} \xi_{i j}}{\xi_{i j} \xi_{i j}}, & m \neq n \\
\inf _{0 \neq\left(\xi_{i j}\right) \in S^{n}} \frac{a_{2}^{i j k \ell} \xi_{k l} \xi_{i j}}{\xi_{i j} \xi_{i j}}, & m=n .
\end{array}\right.
$$

THEOREM 1.2. Assume that $\Gamma_{1}$ is star-shaped with respect to some point $x_{0} \in \Omega_{1}$ and let $\Gamma\left(x_{0}\right), \Sigma\left(x_{0}\right)$ and $R\left(x_{0}\right)$ be as in Theorem 1.1. Let

$$
\begin{aligned}
& \mathcal{R}_{T}=\left\{\left(\mathbf{W}_{1}(T), \mathbf{W}_{2}(T), \dot{\mathbf{W}}_{1}(T), \dot{\mathbf{W}}_{2}(T)\right): \mathbf{U} \in\left[L^{2}(\Sigma)\right]^{m}, \mathbf{U}=0 \text { on } \Sigma \backslash \Sigma\left(x_{0}\right)\right\} . \\
& \text { If }
\end{aligned}
$$$$
\begin{cases}a_{1}^{i j k \ell} \xi_{i j} \xi_{k \ell} \geq a_{2}^{i j k \ell} \xi_{i j} \xi_{k \ell}, & \forall\left(\xi_{i j}\right) \in \mathbb{R}^{m \times n}, m \neq n \\ a_{1}^{i j k \ell} \xi_{i j} \xi_{k \ell} \geq a_{2}^{i j k \ell} \xi_{i j} \xi_{k \ell}, & \forall\left(\xi_{i j}\right) \in S^{n}, m=n,\end{cases}
$$

and if

$$
T>T\left(x_{0}\right)= \begin{cases}\frac{2 R\left(x_{0}\right)}{\sqrt{\left|A_{2}\right|}}, & m \neq n \\ \frac{2 \sqrt{2} R\left(x_{0}\right)}{\sqrt{\left|A_{2}\right|}}, & m=n .\end{cases}
$$

then $\mathcal{R}_{T}=H \times V^{\prime}$ where

$$
\begin{gathered}
H=\left[L^{2}\left(\Omega_{1}\right)\right]^{m} \times\left[L^{2}\left(\Omega_{2}\right)\right]^{m}, \\
V=\left\{\left(\Phi_{1}, \Phi_{2}\right) \in\left[H^{1}\left(\Omega_{1}\right)\right]^{m} \times\left[H^{1}\left(\Omega_{2}\right)\right]^{m}:\left.\Phi_{2}\right|_{\Gamma}=0,\left.\Phi_{1}\right|_{\Gamma_{1}}=\left.\Phi_{2}\right|_{\Gamma_{1}}\right\} .
\end{gathered}
$$

REMARK 1.3. Whether or not the monotonicity condition (1.14) is necessary for exact controllability in dimension $n \geq 2$ is $u n k n o w n$, even in the case of ordinary wave equations considered in Theorem 1.1. It is known that no such condition is necessary for exact boundary controllability of transmission problems in dimension one or, more generally, for exact controllability of 1dimensional networks in $\mathbb{R}^{3}[3$, Chapter IV].

REMARK 1.4. Set

$$
H_{\Gamma}^{1}\left(\Omega_{2}\right)=\left\{\varphi \in H^{1}\left(\Omega_{2}\right):\left.\varphi\right|_{\Gamma}=0\right\} .
$$

The space $V$ is a closed subspace of $\left[H^{1}\left(\Omega_{1}\right)\right]^{m} \times\left[H_{\Gamma}^{1}\left(\Omega_{2}\right)\right]^{m}$ and $V^{\prime}$ may be identified with the restriction to $V$ of elements in the product space $\left[\left(H^{1}\left(\Omega_{1}\right)\right)^{\prime}\right]^{m} \times\left[\left(H_{\Gamma}^{1}\left(\Omega_{2}\right)\right)^{\prime}\right]^{m}$ (cf. [5, Remark 5.1, p. 376].

REMARK 1.5. With only minor modifications in the proof, Theorem 1.2 can be extended to the situation involving $\Omega$ and $p \geq 2$ nested open sets $\omega_{1}, \ldots, \omega_{p}$ with

$$
\bar{\omega}_{i} \subset \omega_{i+1}, \quad i=1, \ldots, p-1, \quad \omega_{p}=\Omega .
$$

Set

$$
\Omega_{1}=\omega_{1}, \quad \Omega_{i}=\omega_{i} \backslash \bar{\omega}_{i-1}, \quad i=1, \ldots, p .
$$

Setting $\Gamma_{i}:=\partial \omega_{i}$, one should assume that $\Gamma_{i}$ is star-shaped with respect to a point $x_{0} \in \Omega_{1}, i=1, \ldots, p-1$, and that the parameters $a_{\alpha}^{i j k \ell}, \alpha=1, \ldots, p$, 
associated with $\Omega_{\alpha}$ satisfy appropriate monotonicity conditions with respect to $\alpha$.

ExamPle 1.6. (Wave equations) Suppose that $m=1$. In this case the system (1.5) may be written

$$
\begin{cases}\ddot{w}_{1}-a_{1}^{j \ell} w_{1, j \ell} & \text { in } Q_{1}, \\ \ddot{w}_{2}-a_{2}^{j \ell} w_{2, j \ell} & \text { in } Q_{2}\end{cases}
$$

where $a_{\alpha}^{j \ell}=a_{\alpha}^{\ell j}$. If we set $A_{\alpha}=\left(a_{\alpha}^{j \ell}\right)$, the condition (1.14) with $m=1<n$ becomes the requirement that the matrix $A_{1}-A_{2}$ be positive semidefinite. The control time $T\left(x_{0}\right)$ is given by $2 R\left(x_{0}\right) / \sqrt{\left|A_{2}\right|}$, where

$$
\left|A_{2}\right|=\inf _{0 \neq \xi \in \mathbb{R}^{n}} \frac{A_{2} \xi \cdot \xi}{|\xi|^{2}} .
$$

In particular, if $A_{\alpha}=a_{\alpha} I$, where $I$ is the identity matrix in $\mathbb{R}^{n}$, we recover Theorem 1.1.

Example 1.7. (Linear isotropic elastodynamics) Here $n=m=3$ and the system of equations is (we do not use summation convention in this example)

$$
\left\{\begin{array}{l}
\rho_{1} \ddot{w}_{1 i}-\mu_{1} \Delta w_{1 i}-\left(\lambda_{1}+\mu_{1}\right) \sum_{j=1}^{3} w_{1 j, j i}=0 \quad \text { in } Q_{1}, \\
\rho_{2} \ddot{w}_{2 i}-\mu_{2} \Delta w_{2 i}-\left(\lambda_{2}+\mu_{2}\right) \sum_{j=1}^{3} w_{2 j, j i}=0 \quad \text { in } Q_{2}, \quad i=1,2,3
\end{array}\right.
$$

where $\rho_{\alpha}$ is the (constant) mass density per unit of reference volume and $\mu_{\alpha}$, $\lambda_{\alpha}$ are the Lamé parameters for the elastic material occupying the region $\Omega_{\alpha}$. We assume that

$$
\mu_{\alpha}>0 \text { and } \lambda_{\alpha} \geq 0
$$

By a change of notation we may assume that $\rho_{1}=\rho_{2}=1$. Then the nonzero coefficients $a_{\alpha}^{i j k \ell}$ are given by

$$
\begin{gathered}
a_{\alpha}^{i i i i}=\lambda_{\alpha}+2 \mu_{\alpha}, \quad i=1,2,3, \\
a_{\alpha}^{i j i j}=a_{\alpha}^{i j j i}=\mu_{\alpha}, \quad a_{\alpha}^{i i j j}=\lambda_{\alpha}, \quad i \neq j=1,2,3,
\end{gathered}
$$

and therefore for $\left(\xi_{i j}\right) \in S^{3}$ we have

$$
\begin{aligned}
\sum a_{\alpha}^{i j k \ell} \xi_{i j} \xi_{k \ell} & =\left(\lambda_{\alpha}+2 \mu_{\alpha}\right) \sum_{i=1}^{3} \xi_{i i}^{2}+\lambda_{\alpha} \sum_{\substack{i, j=1 \\
i \neq j}}^{3} \xi_{i i} \xi_{j j}+\frac{\mu_{\alpha}}{2} \sum_{\substack{i, j=1 \\
i \neq j}}^{3}\left(\xi_{i j}+\xi_{j i}\right)^{2} \\
& =\lambda_{\alpha}\left(\sum_{i=1}^{3} \xi_{i i}\right)^{2}+2 \mu_{\alpha} \sum_{i, j=1}^{3} \xi_{i j}^{2} \geq 2 \mu_{\alpha} \sum_{i, j=1}^{3} \xi_{i j}^{2} .
\end{aligned}
$$

In the same way, the monotonicity condition (1.14) (with strict inequality) will be satisfied if

$$
\mu_{1} \geq \mu_{2} \text { and } \lambda_{1} \geq \lambda_{2} .
$$

The control time $T\left(x_{0}\right)$ is given by $2 \sqrt{2} R\left(x_{0}\right) / \sqrt{\left|A_{2}\right|}=2 R\left(x_{0}\right) / \sqrt{\mu_{2}}$, which is what is expected since the two wave speeds associated with the region $\Omega_{2}$ are $\sqrt{\mu_{2}}$ and $\sqrt{\lambda_{2}+2 \mu_{2}}$.

ESAIM: COCV, , 
Let us briefly recount other works on boundary controllability in problems of transmission or, more generally, in interface problems. These works are for the most part disjoint from the present paper. For one-dimensional interface problems (for example, string or beam equations on one-dimensional graphs in $\mathbb{R}^{3}$ ), one may refer to [3] for a comprehensive analysis of the reachability problem utilizing controls supported at the nodes of the graph. These systems, however, do not generally fall within the framework of the problem (1.5) - (1.8). For higher dimensional systems, one may cite [2], where boundary controllability in transmissions problems for the Reissner-Mindlin system of thin plate theory is considered. This system consists of two coulpled second order equations with constant coefficients but with lower order terms as well, so they too fall outside of the scope of the present work. There are also several works dealing with boundary controllability in problems of transmission or in interface problems for the wave equation in the singular case. If, for example, $\Gamma_{1} \cap \Gamma \neq \emptyset$ in the problem (1.9) - (1.12) above, the solution will generally suffer a loss of regularity in a neighborhood of points belonging to this set. In this case the proof technique for Theorem 1.1 given in [5] fails; that is the reason for the assumption $\bar{\Omega}_{1} \subset \Omega$. In the singular case, we can refer to two recent papers of $S$. Nicaise $[6],[7]$, where the question of exact boundary controllability for the wave equation on two-dimensional networks in $\mathbb{R}^{n}$ is treated. In these papers it is shown, among other things, that a result analogous to Theorem 1.1 may be obtained provided that, in addition to boundary controls, controls are also imposed near the singular vertices. The reader is referred to the cited papers for the precise statements of Nicaise's results. References to other works related to the topic of the present paper may be found in the bibliographies of the books and papers cited above.

\section{A PRIORI ESTIMATES}

Let $H$ and $V$ be as in Theorem 1.2. For $k \geq 0$ set

$$
\mathcal{H}^{k}(\Omega)=\left[H^{k}\left(\Omega_{1}\right)\right]^{m} \times\left[H^{k}\left(\Omega_{2}\right)\right]^{m} .
$$

Let $\Phi:=\left(\Phi_{1}, \Phi_{2}\right), \Psi:=\left(\Psi_{1}, \Psi_{2}\right)$ be elements of $V$ and consider the bilinear form

$$
\sigma(\Phi, \Psi)=\sum_{\alpha=1}^{2} \int_{\Omega_{\alpha}} a_{\alpha}^{i j k \ell} \varphi_{\alpha k, \ell} \psi_{\alpha i, j} d x, \quad \Phi, \Psi \in V .
$$

This form is continuous on $V \times V$ in the topology induced by $\mathcal{H}^{1}(\Omega)$ and is symmetric by virtue of (1.1). If $m \neq n, \sigma$ is also coercive on $V$ because of the ellipticity assumption (1.3) and $\left.\Phi_{2}\right|_{\Gamma}=0$, that is, for some $k>0$,

$$
\sigma(\Phi, \Phi) \geq k\|\Phi\|_{\mathcal{H}^{1}(\Omega)}^{2}, \quad \forall \Phi \in V .
$$

On the other hand, if $m=n$, because of the symmetry assumptions (1.2) we may write

$$
\sigma(\Phi, \Psi)=\sum_{\alpha=1}^{2} \int_{\Omega_{\alpha}} a_{\alpha}^{i j k \ell} \varepsilon_{k \ell}\left(\Phi_{\alpha}\right) \varepsilon_{i j}\left(\Psi_{\alpha}\right) d x
$$


where

$$
\varepsilon_{k \ell}(\Phi)=\frac{1}{2}\left(\varphi_{k, \ell}+\varphi_{\ell, k}\right) .
$$

If $\Phi \in V$ then the functions

$$
\varphi_{i}(x)=\left\{\begin{array}{ll}
\varphi_{1 i}(x), & x \in \Omega_{1} \\
\varphi_{2 i}(x), & x \in \Omega_{2},
\end{array} \quad i=1, \ldots, m\right.
$$

are in $H_{0}^{1}(\Omega)$ and therefore the following Korn's inequality holds [8, Theorem $2.1]$ :

$$
\sum_{\alpha=1}^{2} \int_{\Omega_{\alpha}} \varepsilon_{i j}\left(\Phi_{\alpha}\right) \varepsilon_{i j}\left(\Phi_{\alpha}\right) d x \geq \frac{1}{2} \sum_{\alpha=1}^{2} \int_{\Omega_{\alpha}} \varphi_{\alpha i, j} \varphi_{\alpha i, j} d x .
$$

It follows from (2.1) and (1.4) that

$$
\sigma(\Phi, \Phi) \geq c_{o} \sum_{\alpha=1}^{2} \int_{\Omega_{\alpha}} \varepsilon_{i j}\left(\Phi_{\alpha}\right) \varepsilon_{i j}\left(\Phi_{\alpha}\right) d x \geq k\|\Phi\|_{\mathcal{H}^{1}(\Omega)}^{2}, \quad \forall \Phi \in V,
$$

Therefore in all cases $[\sigma(\Phi, \Phi)]^{1 / 2}$ defines a norm on $V$ equivalent to the one induced by $\mathcal{H}^{1}(\Omega)$. We retopologize $V$ with this norm and let $V^{\prime}$ denote the dual space of $V$. With $H$ identified with its dual space we have the dense and continuous embeddings $V \subset H \subset V^{\prime}$.

Let us consider the homogeneous boundary value problem

$$
\begin{gathered}
\left\{\begin{array}{l}
\ddot{\varphi}_{1 i}-a_{1}^{i j k \ell} \varphi_{1 k, \ell j}=0 \quad \text { in } Q_{1}, \\
\ddot{\varphi}_{2 i}-a_{2}^{i j k \ell} \varphi_{2 k, \ell j}=0 \quad \text { in } Q_{2}, \quad i=1, \ldots, m, \\
\Phi_{2}=0 \quad \text { on } \Sigma,
\end{array}\right. \\
\varphi_{1 i}=\varphi_{2 i}, \quad a_{1}^{i j k \ell} \varphi_{1 k, \ell} \nu_{j}=a_{2}^{i j k \ell} \varphi_{2 k, \ell} \nu_{j} \quad \text { on } \Sigma_{1}, \quad i=1, \ldots, m, \\
\left.\Phi_{\alpha}\right|_{t=0}=\Phi_{\alpha}^{0},\left.\quad \dot{\Phi}_{\alpha}\right|_{t=0}=\Phi_{\alpha}^{1} \quad \text { in } \Omega_{\alpha}, \alpha=1,2 .
\end{gathered}
$$

Assume that $\Phi^{0}:=\left(\Phi_{1}^{0}, \Phi_{2}^{0}\right) \in V, \Phi^{1}:=\left(\Phi_{1}^{1}, \Phi_{2}^{1}\right) \in H$. According to standard variational theory $(2.2)-(2.5)$ has a unique solution in the following sense: there is a unique function $\Phi:=\left(\Phi_{1}, \Phi_{2}\right) \in C([0, T] ; V) \cap$ $C^{1}([0, T] ; H) \cap C^{2}\left([0, T] ; V^{\prime}\right)$ such that

$$
\langle\ddot{\Phi}, \Psi\rangle_{V}+\sigma(\Phi, \Psi)=0, \quad \forall \Psi \in V, 0<t \leq T
$$

and which satisfies $(2.5)$, where $\langle\Phi, \Psi\rangle_{V}$ denotes the pairing between elements $\Phi \in V^{\prime}$ and $\Psi \in V$ in the $V^{\prime}-V$ duality. Furthermore, the mapping $S(t):\left(\Phi^{0}, \Phi^{1}\right) \mapsto(\Phi(t), \dot{\Phi}(t))$ is a defines a unitary group on $V \times H$, that is

$$
\|\Phi(t)\|_{V}^{2}+\|\dot{\Phi}(t)\|_{H}^{2}=\left\|\Phi^{0}\right\|_{V}^{2}+\left\|\Phi^{1}\right\|_{H}^{2}, \quad t \geq 0
$$

Let $A$ denote the canonical isomorphism of $V$ onto $V^{\prime}$, so that $\sigma(\Phi, \Psi)=$ $\langle A \Phi, \Psi\rangle_{V}, \forall \Phi, \Psi \in V$, and set

$$
D(A)=\{\Psi \in V: A \Psi \in H\}, \quad\|\Psi\|_{D(A)}=\|A \Psi\|_{H} .
$$

If $\left(\Phi^{0}, \Phi^{1}\right) \in D(A) \times V$, then $\Phi \in C([0, T] ; D(A)) \cap C^{1}([0, T] ; V) \cap C^{2}([0, T] ; H)$ and $S(t)$ is a unitary group on $D(A) \times V$. Because of our assumptions on the regions $\Omega$ and $\Omega_{1}$, an element $\Phi \in D(A)$ has the regularity $\Phi \in$ ESAIM: COCV, , , 
$\mathcal{H}^{2}(\Omega) \cap V$ and $A \Phi=-\left(a_{1}^{i j k \ell} \varphi_{1 k, j \ell}, a_{2}^{i j k \ell} \varphi_{2 k, j \ell}\right)$. Moreover, $\Phi$ satisfies both of the transmission conditions in (2.4) in the trace sense and the norm $\|\cdot\|_{D(A)}$ is equivalent to that induced on $D(A)$ by $\mathcal{H}^{2}(\Omega)$. These results are standard and may be proved by the usual methods.

Lemma 2.1. Suppose that $\left(\Phi^{0}, \Phi^{1}\right) \in D(A) \times V$, and let $h \in\left[W^{1, \infty}(\Omega)\right]^{n}$. Then the solution of $(2.2)-(2.5)$ satisfies the following identity:

$$
\begin{aligned}
& \sum_{\alpha=1}^{2}\left\{\left.\int_{\Omega_{\alpha}} \dot{\varphi}_{\alpha i}\left(h \cdot \nabla \varphi_{\alpha i}\right) d x\right|_{0} ^{T}\right. \\
&+\frac{1}{2} \int_{Q_{\alpha}}(\operatorname{div} h)\left[\dot{\varphi}_{\alpha i} \dot{\varphi}_{\alpha i}-a_{\alpha}^{i j k \ell} \varphi_{\alpha k, \ell} \varphi_{\alpha i, j}\right] d x d t \\
&\left.\quad+\int_{Q_{\alpha}} a_{\alpha}^{i j k \ell} \varphi_{\alpha k, \ell} \varphi_{\alpha i, p} h_{p, j} d x d t\right\} \\
&=\frac{1}{2} \int_{\Sigma}(h \cdot \nu) a_{2}^{i j k \ell}\left(\frac{\partial \varphi_{2 k}}{\partial \nu}\right)\left(\frac{\partial \varphi_{2 i}}{\partial \nu}\right) \nu_{j} \nu_{\ell} d \Sigma \\
&+\frac{1}{2} \int_{\Sigma_{1}}(h \cdot \nu)\left[\left(a_{1}^{i j k \ell}-a_{2}^{i j k \ell}\right) \varphi_{1 i, j} \varphi_{1 k, \ell}\right. \\
&\left.+a_{2}^{i j k \ell}\left(\frac{\partial \varphi_{1 i}}{\partial \nu}-\frac{\partial \varphi_{2 i}}{\partial \nu}\right)\left(\frac{\partial \varphi_{1 k}}{\partial \nu}-\frac{\partial \varphi_{2 k}}{\partial \nu}\right) \nu_{j} \nu_{\ell}\right] d \Sigma .
\end{aligned}
$$

Proof. The proof utilizes classical multipliers. Form

$$
0=\int_{Q_{\alpha}}\left(\ddot{\varphi}_{\alpha i}-a_{\alpha}^{i j k \ell} \varphi_{\alpha k, \ell j}\right) h \cdot \nabla \varphi_{\alpha i} d x d t
$$

and integrate by parts. One has

$$
\begin{aligned}
\int_{Q_{\alpha}} \ddot{\varphi}_{\alpha i}\left(h \cdot \nabla \varphi_{\alpha i}\right) d x d t & =\left.\int_{\Omega_{\alpha}} \dot{\varphi}_{\alpha i}\left(h \cdot \nabla \varphi_{\alpha i}\right) d x\right|_{0} ^{T} \\
& +\frac{1}{2} \int_{Q_{\alpha}}(\operatorname{div} h) \dot{\varphi}_{\alpha i} \dot{\varphi}_{\alpha i} d x d t-\frac{1}{2} \int_{\Sigma_{\alpha}}(h \cdot \nu) \dot{\varphi}_{\alpha i} \dot{\varphi}_{\alpha i} d \Sigma
\end{aligned}
$$

where $\Sigma_{\alpha}=\partial \Omega_{\alpha} \times(0, T)$ and $\nu$ is the unit normal vector pointing into the exterior of $\Omega_{\alpha}$. In addition,

$$
\begin{array}{r}
\int_{Q_{\alpha}}\left(a_{\alpha}^{i j k \ell} \varphi_{\alpha k, \ell j}\right) h \cdot \nabla \varphi_{\alpha i} d x d t=\int_{\Sigma_{\alpha}} a_{\alpha}^{i j k \ell} \varphi_{\alpha k, \ell} \varphi_{\alpha i, p} h_{p} \nu_{j} d \Sigma \\
-\int_{Q_{\alpha}} a_{\alpha}^{i j k \ell} \varphi_{\alpha k, \ell} \varphi_{\alpha i, p} h_{p, j} d x d t-\int_{Q_{\alpha}} a_{\alpha}^{i j k \ell} \varphi_{\alpha k, \ell} \varphi_{\alpha i, p j} h_{p} d x d t .
\end{array}
$$

From the symmetry property (1.1) we obtain

$$
a_{\alpha}^{i j k \ell} \varphi_{\alpha k, \ell} \varphi_{\alpha i, p j}=\frac{1}{2}\left(a_{\alpha}^{i j k \ell} \varphi_{\alpha k, \ell} \varphi_{\alpha i, j}\right)_{, p}
$$


and therefore

$$
\begin{aligned}
& \int_{Q_{\alpha}}\left(a_{\alpha}^{i j k \ell} \varphi_{\alpha k, \ell j}\right) h \cdot \nabla \varphi_{\alpha i} d x d t \\
& \quad=\int_{\Sigma_{\alpha}} a_{\alpha}^{i j k \ell} \varphi_{\alpha k, \ell} \varphi_{\alpha i, p} h_{p} \nu_{j} d \Sigma-\frac{1}{2} \int_{\Sigma_{\alpha}}(h \cdot \nu) a_{\alpha}^{i j k \ell} \varphi_{\alpha k, \ell} \varphi_{\alpha i, j} d \Sigma \\
& -\int_{Q_{\alpha}} a_{\alpha}^{i j k \ell} \varphi_{\alpha k, \ell} \varphi_{\alpha i, p} h_{p, j} d x d t+\frac{1}{2} \int_{Q_{\alpha}}(\operatorname{div} h) a_{\alpha}^{i j k \ell} \varphi_{\alpha k, \ell} \varphi_{\alpha i, j} d x d t .
\end{aligned}
$$

We then obtain from $(2.8)-(2.10)$ the identity

$$
\begin{aligned}
& \left.\int_{\Omega_{\alpha}} \dot{\varphi}_{\alpha i}\left(h \cdot \nabla \varphi_{\alpha i}\right) d x\right|_{0} ^{T}+\int_{Q_{\alpha}} a_{\alpha}^{i j k \ell} \varphi_{\alpha k, \ell} \varphi_{\alpha i, p} h_{p, j} d x d t \\
& \quad+\frac{1}{2} \int_{Q_{\alpha}}(\operatorname{div} h)\left[\dot{\varphi}_{\alpha i} \dot{\varphi}_{\alpha i}-a_{\alpha}^{i j k \ell} \varphi_{\alpha k, \ell} \varphi_{\alpha i, j}\right] d x d t \\
& =\frac{1}{2} \int_{\Sigma_{\alpha}}(h \cdot \nu)\left(\dot{\varphi}_{\alpha i} \dot{\varphi}_{\alpha i}-a_{\alpha}^{i j k \ell} \varphi_{\alpha k, \ell} \varphi_{\alpha i, j}\right) d \Sigma+\int_{\Sigma_{\alpha}} a_{\alpha}^{i j k \ell} \varphi_{\alpha k, \ell} \varphi_{\alpha i, p} h_{p} \nu_{j} d \Sigma .
\end{aligned}
$$

We sum (2.11) over $\alpha=1,2$, noting the relations

$$
\left.\varphi_{1 i}\right|_{\Sigma_{1}}=\left.\varphi_{2 i}\right|_{\Sigma_{1}},\left.\quad \varphi_{2 i, j}\right|_{\Sigma}=\frac{\partial \varphi_{2 i}}{\partial \nu} \nu_{j}, \quad i=1, \ldots, m ; j=1, \ldots, n
$$

and observing that along the boundary region $\Gamma_{1}$ that is common to $\Omega_{1}$ and $\Omega_{2}$ the unit normal vector pointing out of $\Omega_{2}$ points into $\Omega_{1}$. We obtain

$$
\begin{aligned}
\sum_{\alpha=1}^{2}\left\{\left.\int_{\Omega_{\alpha}} \dot{\varphi}_{\alpha i}\left(h \cdot \nabla \varphi_{\alpha i}\right) d x\right|_{0} ^{T}+\int_{Q_{\alpha}} a_{\alpha}^{i j k \ell} \varphi_{\alpha k, \ell} \varphi_{\alpha i, p} h_{p, j} d x d t\right. \\
\left.+\frac{1}{2} \int_{Q_{\alpha}}(\operatorname{div} h)\left(\dot{\varphi}_{\alpha i} \dot{\varphi}_{\alpha i}-a_{\alpha}^{i j k \ell} \varphi_{\alpha k, \ell} \varphi_{\alpha i, j}\right) d x d t\right\} \\
=\frac{1}{2} \int_{\Sigma}(h \cdot \nu) a_{2}^{i j k \ell}\left(\frac{\partial \varphi_{2 k}}{\partial \nu}\right)\left(\frac{\partial \varphi_{2 i}}{\partial \nu}\right) \nu_{j} \nu_{\ell} d \Sigma \\
+\frac{1}{2} \int_{\Sigma_{1}}(h \cdot \nu)\left(a_{1}^{i j k \ell} \varphi_{1 k, \ell} \varphi_{1 i, j}-a_{2}^{i j k \ell} \varphi_{2 k, \ell} \varphi_{2 i, j}\right) d \Sigma \\
+\int_{\Sigma_{1}}\left(a_{2}^{i j k \ell} \varphi_{2 k, \ell} \varphi_{2 i, p}-a_{1}^{i j k \ell} \varphi_{1 k, \ell} \varphi_{1 i, p}\right) h_{p} \nu_{j} d \Sigma
\end{aligned}
$$

To complete the proof we need to show that the last two integrals in (2.12) agree with the last integral in (2.7).

Consider the last integral in (2.12). By choosing suitable extensions of $\Phi_{1}$ and $\Phi_{2}$ we may assume that $\varphi_{\alpha i} \in H^{2}(\Omega)$ for $\alpha=1,2$ and $i=1, \ldots, n$. We have

$$
\begin{aligned}
& \left(a_{2}^{i j k \ell} \varphi_{2 k, \ell} \varphi_{2 i, p}-a_{1}^{i j k \ell} \varphi_{1 k, \ell} \varphi_{1 i, p}\right) h_{p} \nu_{j} \\
= & a_{2}^{i j k \ell} \varphi_{2 k, \ell} \nu_{j} h_{p}\left(\varphi_{2 i, p}-\varphi_{1 i, p}\right)=(h \cdot \nu) a_{2}^{i j k \ell} \varphi_{2 k, \ell} \nu_{j}\left(\frac{\partial \varphi_{2 i}}{\partial \nu}-\frac{\partial \varphi_{1 i}}{\partial \nu}\right)
\end{aligned}
$$


on $\Sigma_{1}$, since

$\left.\left(\varphi_{2 i, p}-\varphi_{1 i, p}\right)\right|_{\Gamma_{1}}=\left(\frac{\partial \varphi_{2 i}}{\partial \nu}-\frac{\partial \varphi_{1 i}}{\partial \nu}\right) \nu_{p}, \quad i=1, \ldots, m ; p=1, \ldots, n$.

It also follows from (2.14) that on $\Sigma_{1}$

$$
a_{1}^{i j k \ell} \varphi_{1 k, \ell} \varphi_{1 i, j}=a_{1}^{i j k \ell} \varphi_{1 k, \ell}\left[\varphi_{2 i, j}+\left(\frac{\partial \varphi_{1 i}}{\partial \nu}-\frac{\partial \varphi_{2 i}}{\partial \nu}\right) \nu_{j}\right]
$$

and

$$
a_{2}^{i j k \ell} \varphi_{2 k, \ell} \varphi_{2 i, j}=a_{2}^{i j k \ell} \varphi_{2 k, \ell}\left[\varphi_{1 i, j}-\left(\frac{\partial \varphi_{1 i}}{\partial \nu}-\frac{\partial \varphi_{2 i}}{\partial \nu}\right) \nu_{j}\right] .
$$

If the last expression is subtracted from the preceding one we obtain

$$
\begin{aligned}
& a_{1}^{i j k \ell} \varphi_{1 k, \ell} \varphi_{1 i, j}-a_{2}^{i j k \ell} \varphi_{2 k, \ell} \varphi_{2 i, j} \\
= & \left(a_{1}^{i j k \ell}-a_{2}^{i j k \ell}\right) \varphi_{1 k, \ell} \varphi_{2 i, j}+2 a_{2}^{i j k \ell} \varphi_{2 k, \ell} \nu_{j}\left(\frac{\partial \varphi_{1 i}}{\partial \nu}-\frac{\partial \varphi_{2 i}}{\partial \nu}\right) \quad \text { on } \Sigma_{1},
\end{aligned}
$$

where we have used the symmetry property (1.1) and the second transmission condition in (2.4). It follows from (2.13) and (2.15) that

$$
\begin{aligned}
\frac{1}{2} \int_{\Sigma_{1}}(h \cdot \nu) & \left(a_{1}^{i j k \ell} \varphi_{1 k, \ell} \varphi_{1 i, j}-a_{2}^{i j k \ell} \varphi_{2 k, \ell} \varphi_{2 i, j}\right) d \Sigma \\
& +\int_{\Sigma_{1}}\left(a_{2}^{i j k \ell} \varphi_{2 k, \ell} \varphi_{2 i, p} h_{p} \nu_{j}-a_{1}^{i j k \ell} \varphi_{1 k, \ell} \varphi_{1 i, p} h_{p} \nu_{j}\right) d \Sigma \\
& =\frac{1}{2} \int_{\Sigma_{1}}(h \cdot \nu)\left(a_{1}^{i j k \ell}-a_{2}^{i j k \ell}\right) \varphi_{1 k, \ell} \varphi_{2 i, j} d \Sigma
\end{aligned}
$$

We use (2.14) once again to obtain on $\Sigma_{1}$

$$
\begin{aligned}
& \left(a_{1}^{i j k \ell}-a_{2}^{i j k \ell}\right) \varphi_{1 k, \ell} \varphi_{2 i, j} \\
& =\left(a_{1}^{i j k \ell}-a_{2}^{i j k \ell}\right) \varphi_{1 k, \ell} \varphi_{1 i, j}+\left(a_{1}^{i j k \ell}-a_{2}^{i j k \ell}\right) \varphi_{1 k, \ell} \nu_{j}\left(\frac{\partial \varphi_{2 i}}{\partial \nu}-\frac{\partial \varphi_{1 i}}{\partial \nu}\right) \\
& =\left(a_{1}^{i j k \ell}-a_{2}^{i j k \ell}\right) \varphi_{1 k, \ell} \varphi_{1 i, j}+a_{2}^{i j k \ell} \varphi_{2 k, \ell} \nu_{j}\left(\frac{\partial \varphi_{2 i}}{\partial \nu}-\frac{\partial \varphi_{1 i}}{\partial \nu}\right) \\
& \quad-a_{2}^{i j k \ell} \varphi_{1 k, \ell} \nu_{j}\left(\frac{\partial \varphi_{2 i}}{\partial \nu}-\frac{\partial \varphi_{1 i}}{\partial \nu}\right) \\
& =\left(a_{1}^{i j k \ell}-a_{2}^{i j k \ell}\right) \varphi_{1 k, \ell} \varphi_{1 i, j}+a_{2}^{i j k \ell}\left(\varphi_{2 k, \ell}-\varphi_{1 k, \ell}\right) \nu_{j}\left(\frac{\partial \varphi_{2 i}}{\partial \nu}-\frac{\partial \varphi_{1 i}}{\partial \nu}\right) \\
& =\left(a_{1}^{i j k \ell}-a_{2}^{i j k \ell}\right) \varphi_{1 k, \ell} \varphi_{1 i, j}+a_{2}^{i j k \ell}\left(\frac{\partial \varphi_{2 k}}{\partial \nu}-\frac{\partial \varphi_{1 k}}{\partial \nu}\right)\left(\frac{\partial \varphi_{2 i}}{\partial \nu}-\frac{\partial \varphi_{1 i}}{\partial \nu}\right) \nu_{j} \nu_{\ell} .
\end{aligned}
$$

Now substitute (2.17) into (2.16).

Theorem 2.2. Let $\left(\Phi^{0}, \Phi^{1}\right) \in V \times H$. Then the solution of $(2.2)-(2.5)$ satisfies

$$
\int_{\Sigma} a_{2}^{i j k \ell}\left(\frac{\partial \varphi_{2 k}}{\partial \nu}\right)\left(\frac{\partial \varphi_{2 i}}{\partial \nu}\right) \nu_{j} \nu_{\ell} d \Sigma \leq C(T+1)\left(\left\|\Phi^{0}\right\|_{V}^{2}+\left\|\Phi^{1}\right\|_{H}^{2}\right)
$$

for some constant $C$. 
Proof. By density it suffices to prove $(2.18)$ for $\left(\Phi^{0}, \Phi^{1}\right) \in D(A) \times V$. Since $\Gamma$ and $\Gamma_{1}$ are smooth, there is a vector field $h \in\left[C^{\infty}(\bar{\Omega})\right]^{n}$ such that $h=\nu$ on $\Gamma$ and $h=0$ on $\Gamma_{1}$ (see Komornik [1, Lemma 2.1]). We utilize this vector field in (2.7) and observe that the left side of (2.7) is bounded above in absolute value by

$$
\begin{aligned}
& C_{o}\left(\|\dot{\Phi}(T)\|_{H}^{2}+\|\Phi(T)\|_{V}^{2}+\left\|\Phi^{1}\right\|_{H}^{2}+\left\|\Phi^{0}\right\|_{V}^{2}\right)+C_{1} T \sup _{[0, T]}\left(\|\Phi(t)\|_{V}^{2}+\|\dot{\Phi}(t)\|_{H}^{2}\right) \\
& \leq C(T+1)\left(\left\|\Phi^{1}\right\|_{H}^{2}+\left\|\Phi^{0}\right\|_{V}^{2}\right)
\end{aligned}
$$

in view of (2.6).

REMARK 2.3. If $m \neq n$ is follows from (2.18) and (1.3) that

$$
\frac{\partial \varphi_{2 i}}{\partial \nu} \in L^{2}(\Sigma), \quad i=1, \ldots, m \text {. }
$$

If $m=n$, then (2.18) and (1.4) imply that

$$
\frac{\partial \varphi_{2 i}}{\partial \nu} \nu_{j}+\frac{\partial \varphi_{2 j}}{\partial \nu} \nu_{i} \in L^{2}(\Sigma), \quad i, j=1, \ldots, n,
$$

so, in particular, $\nu_{i} \partial \varphi_{2 i} / \partial \nu \in L^{2}(\Sigma)$ for each $i=1, \ldots, n$. Since

$$
\sum_{i, j=1}^{n}\left(\frac{\partial \varphi_{2 i}}{\partial \nu} \nu_{j}+\frac{\partial \varphi_{2 j}}{\partial \nu} \nu_{i}\right)^{2}=2 \sum_{i=1}^{n}\left(\frac{\partial \varphi_{2 i}}{\partial \nu}\right)^{2}+2 \sum_{i, j=1}^{n} \nu_{i} \nu_{j} \frac{\partial \varphi_{2 i}}{\partial \nu} \frac{\partial \varphi_{2 j}}{\partial \nu}
$$

and the second sum is in $L^{1}(\Sigma)$, it follows that (2.19) holds true also in this case.

THEOREM 2.4. Assume that $\Gamma_{1}$ is star-shaped with respect to some point $x_{0} \in \Omega_{1}$ and suppose that the coefficients $a_{\alpha}^{i j k \ell}$ satisfy (1.14). Let $\left(\Phi^{0}, \Phi^{1}\right) \in$ $V \times H$. Then the solution of (2.2) - (2.5) satisfies

$$
\left(T-T\left(x_{0}\right)\right)\left(\left\|\Phi^{1}\right\|_{H}^{2}+\left\|\Phi^{0}\right\|_{V}^{2}\right) \leq \int_{\Sigma\left(x_{0}\right)}(h \cdot \nu) a_{2}^{i j k \ell}\left(\frac{\partial \varphi_{2 k}}{\partial \nu}\right)\left(\frac{\partial \varphi_{2 i}}{\partial \nu}\right) \nu_{j} \nu_{\ell} d \Sigma,
$$

where $T\left(x_{0}\right)$ is given by (1.15) and $h(x)=x-x_{0}$.

Proof. Because of (2.18) it suffices to prove $(2.20)$ for $\left(\Phi^{0}, \Phi^{1}\right) \in D(A) \times V$. We apply (2.7) with $h(x)=x-x_{0}$. Then $h \cdot \nu \leq 0$ on $\Gamma_{1}$. On the other hand, assumption (1.14) and the standing assumption (1.1) (or (1.2)) imply that

$$
\left(a_{1}^{i j k \ell}-a_{2}^{i j k \ell}\right) \varphi_{1 i, j} \varphi_{1 k, \ell}+a_{2}^{i j k \ell}\left(\frac{\partial \varphi_{1 i}}{\partial \nu}-\frac{\partial \varphi_{2 i}}{\partial \nu}\right)\left(\frac{\partial \varphi_{1 k}}{\partial \nu}-\frac{\partial \varphi_{2 k}}{\partial \nu}\right) \nu_{j} \nu_{\ell} \geq 0 .
$$

Therefore

$$
\begin{aligned}
& \sum_{\alpha=1}^{2}\left\{\left.\int_{\Omega_{\alpha}} \dot{\varphi}_{\alpha i}\left(h \cdot \nabla \varphi_{\alpha i}\right) d x\right|_{0} ^{T}+\frac{n}{2} \int_{Q_{\alpha}} \dot{\varphi}_{\alpha i} \dot{\varphi}_{\alpha i} d x d t\right. \\
& \left.+\left(1-\frac{n}{2}\right) \int_{Q_{\alpha}} a_{\alpha}^{i j k \ell} \varphi_{\alpha k, \ell} \varphi_{\alpha, j} d x d t+\int_{Q_{\alpha}} a_{\alpha}^{i j k \ell} \varphi_{\alpha k, \ell} \varphi_{\alpha i, p} h_{p, j} d x d t\right\} \\
& \quad \leq \frac{1}{2} \int_{\Sigma\left(x_{0}\right)}(h \cdot \nu) a_{2}^{i j k \ell}\left(\frac{\partial \varphi_{2 k}}{\partial \nu}\right)\left(\frac{\partial \varphi_{2 i}}{\partial \nu}\right) \nu_{j} \nu_{\ell} d \Sigma .
\end{aligned}
$$


Now form

$$
0=\sum_{\alpha=1}^{2}\left\{\int_{Q_{\alpha}}\left(\ddot{\varphi}_{\alpha i}-a_{\alpha}^{i j k \ell} \varphi_{\alpha k, \ell j}\right) \varphi_{\alpha i} d x d t\right\}
$$

and integrate by parts to obtain

$$
\begin{aligned}
\sum_{\alpha=1}^{2}\left\{\left.\int_{\Omega_{\alpha}} \dot{\varphi}_{\alpha i} \varphi_{\alpha i}\right|_{0} ^{T}\right. & \left.-\int_{Q_{\alpha}}\left(\dot{\varphi}_{\alpha i} \dot{\varphi}_{\alpha i}-a_{\alpha}^{i j k \ell} \varphi_{\alpha k, \ell} \varphi_{\alpha i, j}\right) d x d t\right\} \\
& =\int_{\Sigma_{1}}\left(a_{2}^{i j k \ell} \varphi_{2 k, \ell} \varphi_{2 i} \nu_{j}-a_{1}^{i j k \ell} \varphi_{1 k, \ell} \varphi_{1 i} \nu_{j}\right) d \Sigma
\end{aligned}
$$

The right side vanishes because of the transmission conditions (2.4). Multiply $(2.22)$ by $(n-1) / 2$ and add the result from (2.21) to obtain

$$
\begin{aligned}
\sum_{\alpha=1}^{2}\left\{\int_{\Omega_{\alpha}} \dot{\varphi}_{\alpha i}(h\right. & \left.\cdot \nabla \varphi_{\alpha i}+\frac{n-1}{2} \varphi_{\alpha i}\right)\left.d x\right|_{0} ^{T} \\
+ & \left.\frac{1}{2} \int_{Q_{\alpha}}\left(\dot{\varphi}_{\alpha i} \dot{\varphi}_{\alpha i}+a_{\alpha}^{i j k \ell} \varphi_{\alpha k, \ell} \varphi_{\alpha, j}\right) d x d t\right\} \\
& \leq \frac{1}{2} \int_{\Sigma\left(x_{0}\right)}(h \cdot \nu) a_{2}^{i j k \ell}\left(\frac{\partial \varphi_{2 k}}{\partial \nu}\right)\left(\frac{\partial \varphi_{2 i}}{\partial \nu}\right) \nu_{j} \nu_{\ell} d \Sigma
\end{aligned}
$$

One has

$$
\sum_{\alpha=1}^{2} \int_{Q_{\alpha}}\left(\dot{\varphi}_{\alpha i} \dot{\varphi}_{\alpha i}+a_{\alpha}^{i j k \ell} \varphi_{\alpha k, \ell} \varphi_{\alpha, j}\right) d x d t=T\left(\left\|\Phi^{0}\right\|_{V}^{2}+\left\|\Phi^{1}\right\|_{H}^{2}\right)
$$

and (dropping for the moment summation convention over repeated Roman indices)

$$
\begin{aligned}
& \left|\sum_{\alpha=1}^{2} \sum_{i=1}^{m} \int_{\Omega_{\alpha}} \dot{\varphi}_{\alpha i}\left(h \cdot \nabla \varphi_{\alpha i}+\frac{n-1}{2} \varphi_{\alpha i}\right) d x\right| \\
& \quad \leq \frac{1}{2} \sum_{\alpha=1}^{2} \sum_{i=1}^{m}\left\{K \int_{\Omega_{\alpha}} \dot{\varphi}_{\alpha i}^{2} d x+\frac{1}{4 K} \int_{\Omega_{\alpha}}\left(2 h \cdot \nabla \varphi_{\alpha i}+(n-1) \varphi_{\alpha i}\right)^{2} d x\right\}
\end{aligned}
$$

for any $K>0$. From Komornik [1, Lemma 3.2] we have

$$
\int_{\Omega_{\alpha}}\left(2 h \cdot \nabla \varphi_{\alpha i}+(n-1) \varphi_{\alpha i}\right)^{2} d x \leq 2(n-1) \int_{\Gamma_{\alpha}}(h \cdot \nu) \varphi_{\alpha i}^{2} d \Sigma+4 \int_{\Omega_{\alpha}}\left(h \cdot \nabla \varphi_{\alpha i}\right)^{2} d x .
$$

Hence

$$
\begin{aligned}
\mid \sum_{\alpha=1}^{2} \sum_{i=1}^{m} \int_{\Omega_{\alpha}} \dot{\varphi}_{\alpha i}(h & \left.\cdot \nabla \varphi_{\alpha i}+\frac{n-1}{2} \varphi_{\alpha i}\right) d x \mid \\
& \leq \frac{1}{2} \sum_{\alpha=1}^{2} \sum_{i=1}^{m}\left\{K \int_{\Omega_{\alpha}} \dot{\varphi}_{\alpha i}^{2} d x+\frac{1}{K} \int_{\Omega_{\alpha}}\left(h \cdot \nabla \varphi_{\alpha i}\right)^{2} d x\right\}
\end{aligned}
$$


where we have utilized (2.3) and (2.4). Now choose $K=K\left(x_{0}\right)$ to be a constant such that (returning to summation convention over repeated Roman indices)

$$
\sum_{\alpha=1}^{2} \int_{\Omega_{\alpha}}\left(h \cdot \nabla \varphi_{\alpha i}\right)\left(h \cdot \nabla \varphi_{\alpha i}\right) d x \leq K^{2} \sum_{\alpha=1}^{2} \int_{\Omega_{\alpha}} a_{\alpha}^{i j k \ell} \varphi_{\alpha k, l} \varphi_{\alpha i, j} d x, \quad \forall \Phi \in V .
$$

We then obtain

$$
\begin{aligned}
& \left|\sum_{\alpha=1}^{2} \int_{\Omega_{\alpha}} \dot{\varphi}_{\alpha i}\left(h \cdot \nabla \varphi_{\alpha i}+\frac{n-1}{2} \varphi_{\alpha i}\right) d x\right| \\
& \leq \frac{K}{2} \sum_{\alpha=1}^{2} \int_{\Omega_{\alpha}}\left(\dot{\varphi}_{\alpha i} \dot{\varphi}_{\alpha i}+a_{\alpha}^{i j k \ell} \varphi_{\alpha k, \ell} \varphi_{\alpha i, j}\right) d x=\frac{K}{2}\left(\left\|\Phi^{0}\right\|_{V}^{2}+\left\|\Phi^{1}\right\|_{H}^{2}\right) .
\end{aligned}
$$

Use of (2.24) and (2.25) in (2.23) yields the inequality

$$
(T-2 K)\left(\left\|\Phi^{0}\right\|_{V}^{2}+\left\|\Phi^{1}\right\|_{H}^{2}\right) \leq \int_{\Sigma\left(x_{0}\right)}(h \cdot \nu) a_{2}^{i j k \ell}\left(\frac{\partial \varphi_{2 k}}{\partial \nu}\right)\left(\frac{\partial \varphi_{2 i}}{\partial \nu}\right) \nu_{j} \nu_{\ell} d \Sigma .
$$

One may choose

$$
K= \begin{cases}\frac{R\left(x_{0}\right)}{\sqrt{\left|A_{2}\right|}}, & m \neq n \\ \frac{\sqrt{2} R\left(x_{0}\right)}{\sqrt{\left|A_{2}\right|}}, & m=n .\end{cases}
$$

In fact, if $m \neq n$ one has

$$
\begin{aligned}
& \sum_{\alpha=1}^{2} \int_{\Omega_{\alpha}}\left(h \cdot \nabla \varphi_{\alpha i}\right)\left(h \cdot \nabla \varphi_{\alpha i}\right) d x \leq\left(R\left(x_{0}\right)\right)^{2} \sum_{\alpha=1}^{2} \int_{\Omega_{\alpha}} \varphi_{\alpha i, j} \varphi_{\alpha i, j} d x \\
\leq & \frac{\left(R\left(x_{0}\right)\right)^{2}}{\left|A_{2}\right|} \sum_{\alpha=1}^{2} \int_{\Omega_{\alpha}} a_{2}^{i j k l} \varphi_{\alpha k, \ell} \varphi_{\alpha i, j} d x \leq \frac{\left(R\left(x_{0}\right)\right)^{2}}{\left|A_{2}\right|} \sum_{\alpha=1}^{2} \int_{\Omega_{\alpha}} a_{\alpha}^{i j k l} \varphi_{\alpha k, \ell} \varphi_{\alpha i, j} d x,
\end{aligned}
$$

while if $m=n$ we have from (2.1)

$$
\begin{aligned}
\sum_{\alpha=1}^{2} \int_{\Omega_{\alpha}}\left(h \cdot \nabla \varphi_{\alpha i}\right)\left(h \cdot \nabla \varphi_{\alpha i}\right) d x & \leq 2\left(R\left(x_{0}\right)\right)^{2} \sum_{\alpha=1}^{2} \int_{\Omega_{\alpha}} \varepsilon_{i j}\left(\Phi_{\alpha}\right) \varepsilon_{i j}\left(\Phi_{\alpha}\right) d x \\
& \leq \frac{2\left(R\left(x_{0}\right)\right)^{2}}{\left|A_{2}\right|} \sum_{\alpha=1}^{2} \int_{\Omega_{\alpha}} a_{2}^{i j k \ell} \varepsilon_{k l}\left(\Phi_{\alpha}\right) \varepsilon_{i j}\left(\Phi_{\alpha}\right) d x \\
& \leq \frac{2\left(R\left(x_{0}\right)\right)^{2}}{\left|A_{2}\right|} \sum_{\alpha=1}^{2} \int_{\Omega_{\alpha}} a_{\alpha}^{i j k \ell} \varepsilon_{k l}\left(\Phi_{\alpha}\right) \varepsilon_{i j}\left(\Phi_{\alpha}\right) d x \\
& =\frac{2\left(R\left(x_{0}\right)\right)^{2}}{\left|A_{2}\right|} \sum_{\alpha=1}^{2} \int_{\Omega_{\alpha}} a_{\alpha}^{i j k \ell} \varphi_{\alpha k, \ell} \varphi_{\alpha i, j} d x
\end{aligned}
$$




\section{Proof of Theorem 1.2}

Set

$$
\mathcal{U}=\left\{\mathbf{U} \in\left[L^{2}(\Sigma)\right]^{m}: \mathbf{U}=0 \text { on } \Sigma \backslash \Sigma\left(x_{0}\right)\right\} .
$$

We first make precise the sense in which (1.5) - (1.8) is solvable when $\mathbf{U} \in \mathcal{U}$. Proceeding formally for the moment, let $\Phi \in D(A)$ and form

$$
0=\sum_{\alpha=1}^{2} \int_{\Omega_{\alpha}}\left(\ddot{w}_{\alpha i}-a_{\alpha}^{i j k \ell} w_{\alpha k, \ell j}\right) \varphi_{\alpha i} d x .
$$

If we integrate by parts and use (1.1) and properties of $\Phi$ we obtain

$$
\begin{aligned}
0=\sum_{\alpha=1}^{2}\left\{\int_{\Omega_{\alpha}}\left(\ddot{w}_{\alpha i} \varphi_{\alpha i} d x-\int_{\Omega_{\alpha}} a_{\alpha}^{i j k \ell} \varphi_{\alpha k, \ell j} w_{\alpha i} d x\right\}\right. \\
=-\int_{\Gamma\left(x_{0}\right)} a_{2}^{i j k \ell} \varphi_{2 k, \ell} \nu_{j} u_{i} d \Gamma, \quad \mathbf{U}=\left(\begin{array}{c}
u_{1} \\
\vdots \\
u_{m}
\end{array}\right) .
\end{aligned}
$$

The last equation is the same as

$$
(\ddot{\mathbf{W}}, \Phi)_{H}+(\mathbf{W}, A \Phi)_{H}=-\int_{\Gamma\left(x_{0}\right)} a_{2}^{i j k \ell} \varphi_{2 k, \ell} \nu_{j} u_{i} d \Gamma .
$$

Let $\Psi \in H$ and set $\Phi=A^{-1} \Psi$. One has

$$
\left|\int_{\Gamma\left(x_{0}\right)} a_{2}^{i j k \ell} \varphi_{2 k, \ell} \nu_{j} u_{i} d \Gamma\right| \leq C\|\mathbf{U}\| \mathcal{U}\|\Phi\|_{D(A)}=C\|\mathbf{U}\|_{\mathcal{U}}\|\Psi\|_{H}
$$

and therefore there is a $B \in \mathcal{L}(\mathcal{U}, H)$ such that

$$
-\int_{\Gamma\left(x_{0}\right)} a_{2}^{i j k \ell} \varphi_{2 k, \ell} \nu_{j} u_{k} d \Gamma=(B \mathbf{U}, \Psi)_{H}, \quad \forall \Psi \in H
$$

Then (3.1) may be written

$$
\left(\ddot{\mathbf{W}}, A^{-1} \Psi\right)_{H}+(\mathbf{W}, \Psi)_{H}=(B \mathbf{U}, \Psi)_{H}, \quad \forall \Psi \in H,
$$

which is the same as

$$
\ddot{\mathbf{W}}+A \mathbf{W}=A B \mathbf{U} \text { in }(D(A))^{\prime},
$$

where $(D(A))^{\prime}$ is the dual of $D(A)$ with respect to $H$ and $A$ is an isomorphism of $H$ onto $(D(A))^{\prime}$ defined by

$$
\langle A \Psi, \Phi\rangle_{D(A)}=(\Psi, A \Phi)_{H}, \quad \forall \Psi \in H, \Phi \in D(A) .
$$

Since $A B \mathbf{U} \in L^{2}\left(0, T ;(D(A))^{\prime}\right)$ it follows that given initial data

$$
\mathbf{W}(0)=\mathbf{W}^{0} \in V^{\prime}, \quad \dot{\mathbf{W}}(0)=\mathbf{W}^{1} \in(D(A))^{\prime},
$$

the problem $(3.2),(3.3)$ has a unique solution with $\mathbf{W} \in C\left([0, T] ; V^{\prime}\right), \dot{W} \in$ $C\left([0, T] ;(D(A))^{\prime}\right)$. Further, because of Theorem 2.1 and utilizing a "lifting theorem" of Lasiecka and Triggiani [4] we have that

$$
\left(\mathbf{W}^{0}, \mathbf{W}^{1}\right) \in H \times V^{\prime} \Longrightarrow \mathbf{W} \in C([0, T] ; H) \cap C^{1}\left([0, T] ; V^{\prime}\right)
$$

and, moreover,

$$
\|(\mathbf{W}, \dot{\mathbf{W}})\|_{C\left([0, T] ; H \times V^{\prime}\right)} \leq C_{T}\left[\left\|\left(\mathbf{W}^{0}, \mathbf{W}^{1}\right)\right\|_{H \times V^{\prime}}+\underset{\text { ESAIM: CoCv, }, ~}{\left.\|\mathbf{U}\|_{\mathcal{U}}\right] .}\right.
$$


In particular, the problem (1.5) - (1.8) has a unique solution in the sense of (3.2) and

$$
\mathbf{W}(0)=0, \quad \dot{\mathbf{W}}(0)=0 .
$$

To complete the proof we apply the Hilbert uniqueness method [5]. Let $\Phi=\left(\Phi_{1}, \Phi_{2}\right)$ be the solution of the problem

$$
\begin{aligned}
& \begin{cases}\ddot{\varphi}_{1 i}-a_{1}^{i j k \ell} \varphi_{1 k, \ell j}=0 & \text { in } Q_{1}, \\
\ddot{\varphi}_{2 i}-a_{2}^{i j k \ell} \varphi_{2 k, \ell j}=0 & \text { in } Q_{2}, \quad i=1, \ldots, m,\end{cases} \\
& \Phi_{2}=0 \quad \text { on } \Sigma, \\
& \varphi_{1 i}=\varphi_{2 i}, \quad a_{1}^{i j k \ell} \varphi_{1 k, \ell} \nu_{j}=a_{2}^{i j k \ell} \varphi_{2 k, \ell} \nu_{j} \quad \text { on } \Sigma_{1}, \quad i=1, \ldots, m \text {, } \\
& \left.\Phi\right|_{t=T}=\Phi^{0} \in V,\left.\quad \dot{\Phi}\right|_{t=T}=\Phi^{1} \in H .
\end{aligned}
$$

According to Theorems 2.1 and 2.2 , for $T>T\left(x_{0}\right)$ one may define on $V \times H$ an equivalent norm $\|\cdot\|_{F}$ by

$$
\left\|\left(\Phi^{0}, \Phi^{1}\right)\right\|_{F}=\left[\int_{\Sigma\left(x_{0}\right)} a_{2}^{i j k \ell}\left(\frac{\partial \varphi_{2 k}}{\partial \nu}\right)\left(\frac{\partial \varphi_{2 i}}{\partial \nu}\right) \nu_{j} \nu_{\ell} d \Sigma\right]^{1 / 2}
$$

Now let $\mathbf{W}=\left(\mathbf{W}_{1}, \mathbf{W}_{2}\right)$ be the solution of $(1.5)-(1.8)$ in which we choose

$$
\mathbf{U}= \begin{cases}-\left.\frac{\partial \Phi_{2}}{\partial \nu}\right|_{\Sigma} & \text { on } \Sigma\left(x_{0}\right), \\ 0 & \text { on } \Sigma \backslash \Sigma\left(x_{0}\right) .\end{cases}
$$

From

$$
0=\sum_{\alpha=1}^{2} \int_{Q_{\alpha}}\left(\ddot{w}_{\alpha i}-a_{\alpha}^{i j k \ell} w_{\alpha k, \ell j}\right) \varphi_{\alpha i} d x
$$

we obtain by integration by parts

$$
0=\left\langle\dot{\mathbf{W}}(T), \Phi^{0}\right\rangle_{V}-\left(\mathbf{W}(T), \Phi^{1}\right)_{H}-\int_{\Sigma\left(x_{0}\right)} a_{2}^{i j k \ell} \varphi_{2 k, \ell} \nu_{j}\left(\frac{\partial \varphi_{2 i}}{\partial \nu}\right) d \Sigma,
$$

which may be rewritten

$$
\begin{aligned}
\langle(\dot{\mathbf{W}}(T),-\mathbf{W}(T)) & \left.,\left(\Phi^{0}, \Phi^{1}\right)\right\rangle_{V \times H} \\
& =\int_{\Sigma\left(x_{0}\right)} a_{2}^{i j k \ell}\left(\frac{\partial \varphi_{2 k}}{\partial \nu}\right)\left(\frac{\partial \varphi_{2 i}}{\partial \nu}\right) \nu_{j} \nu_{\ell} d \Sigma=\left\|\left(\Phi^{0}, \Phi^{1}\right)\right\|_{F}^{2} .
\end{aligned}
$$

Therefore for $T>T\left(x_{0}\right)$ the map $\Lambda:\left(\Phi^{0}, \Phi^{1}\right) \mapsto(\dot{\mathbf{W}}(T),-\mathbf{W}(T))$ is the canonical isomorphism of $F$ onto $F^{\prime}=V^{\prime} \times H$. Hence, given any $\left(\mathbf{W}^{1},-\mathbf{W}^{0}\right) \in V^{\prime} \times H$ one may choose $\left(\Phi^{0}, \Phi^{1}\right) \in V \times H$ such that

$$
\Lambda\left(\Phi^{0}, \Phi^{1}\right)=\left(\mathbf{W}^{1},-\mathbf{W}^{0}\right),
$$

that is,

$$
\mathbf{W}(T)=\mathbf{W}^{0}, \quad \dot{\mathbf{W}}(T)=\mathbf{W}^{1} .
$$

This proves that $\mathcal{R}_{T}=H \times V^{\prime}$ if $T>T\left(x_{0}\right)$.

ESAIM: COCV, , , 


\section{REFERENCES}

[1] V. Komornik: Exact Controllability and Stabilization: The Multiplier Method, Masson, Paris, 1994.

[2] J. E. Lagnese: Boundary controllability in transmission problems for thin plates, in Differential Equations, Dynamical Systems, and Control Science, K. D. Elworthy, W. N. Everitt and E. B. Lee, Eds., Lecture Notes in Pure and Applied Mathematics, vol. 152, Marcel Dekker, New York, 1994, 641-658.

[3] J. Lagnese, G. Leugering, G. Schmidt: Modeling, Analysis and Control of Dynamic Elastic Multi-Link Structures, Birkhäuser, Boston, 1994.

[4] I. Lasiecka, R. Triggiani: A lifting theorem for the time regularity of solutions of abstract equations with unbounded operators and applications to hyperbolic equations, Proc. AMS 104 (1988), 745-755.

[5] J.-L. Lions: Contrôlabilité Exacte, Perturbations et Stabilisation de Systèmes Distribués: Tome 1, Contrôlabilité Exacte, Coll. RMA, vol. 8, Masson, Paris, 1988.

[6] S. Nicaise: Boundary exact controllability of interface problems with singularities I: addition of the coefficients of singularities, SIAM J. Control \&\& Opt. 34 (1996), 15121532 .

[7] S. Nicaise: Boundary exact controllability of interface problems with singularities II: addition of internal controls, SIAM J. Control \& Opt. 35 (1997), 585-603.

[8] O. A. Oleinik, A. S. Shamaev, G. A. Yosifian: Mathematical Problems in Elasticity and Homogenization, Studies in Mathematics and Its Applications, vol. 26, North-Holland, Amsterdam, 1992. 